\title{
Sexual dysfunction in cervical dystonia and blepharospasm
}

This article was published in the following Dove Press journal:

Neuropsychiatric Disease and Treatment

\author{
M Marek' \\ M Grobe-Einsler' \\ JR Bedarf ${ }^{\prime}$ \\ B Wabbels ${ }^{2}$ \\ S Paus'
}

'Department of Neurology, University of Bonn, Bonn, Germany; ${ }^{2}$ Department of Ophthalmology, University of Bonn, Bonn, Germany
Correspondence: S Paus

Department of Neurology, University of Bonn, Sigmund-Freud-Str. 25, Bonn 53105, Germany

Emailspaus@uni-bonn.de
Background: Sexual dysfunction is a frequent, yet underrated, symptom of neurological disease. While knowledge of non-motor comorbidity in focal dystonia is growing rapidly, there is no information on the prevalence of sexual dysfunction in cervical dystonia (CD) or blepharospasm (BL).

Methods: In this controlled study, we examined sexual dysfunction in 65 patients with $\mathrm{CD}$ and 54 patients with BL by the Arizona Sexual Experience Scale, a validated self-rating scale.

Results: Sexual dysfunction was significantly higher in CD patients $(45 \%)$ than in controls (24\%), and frequent in BL (39\%). Interestingly, variables of dystonia such as disease duration or severity did not influence sexuality; yet, $23 \%$ of CD patients ascribed worsening of their sexual life to dystonia. Symptoms of depression were identified as the most important predictors for sexual dysfunction, followed by age, and personal status (single).

Conclusion: Our observations establish sexual dysfunction as a frequent non-motor symptom in $\mathrm{CD}$ and $\mathrm{BL}$ that is perceived as a burden. It should be considered when investigating patients with adult-onset focal dystonia.

Keywords: dystonia, cervical dystonia, blepharospasm, sexual dysfunction, depression

\section{Background}

Sexual dysfunction is a frequent and troublesome symptom of various neurological diseases and their treatments, considerably impairing quality of life. ${ }^{1}$ Nevertheless, patient care and scientific studies often omit sexual issues. In movement disorders, knowledge on sexual dysfunction in Parkinson's disease (PD) is growing, ${ }^{2}$ yet is scarce in focal dystonia, a group of chronic diseases without causal therapeutic options. Today, perception of focal dystonia shifts from that of an isolated movement disorder to a disease with important non-motor comorbidity, foremost mood disturbances. ${ }^{3,4}$ Furthermore, anxiety, ${ }^{3,5}$ social embarrassment and social phobia, ${ }^{6}$ and psychosocial effects such as stigmatization ${ }^{7}$ are common in cervical dystonia (CD) and blepharospasm (BL), often independent from the motor severity of dystonia. As the relationship of mental health and sexual functioning is multilayered and mutual, ${ }^{1,8}$ it is surprising that, until now, only one study has examined sexual well-being in patients suffering from dystonia. ${ }^{9}$ In that pilot examination in $30 \mathrm{BL}$ and $30 \mathrm{CD}$ patients, depression and anxiety were identified as predictors of sexual dysfunction. ${ }^{9}$

In the epidemiological case-control study presented here, we aimed to evaluate prevalence, risk factors, and associated clinical variables of sexual impairment in focal dystonias by examination of larger samples of CD and BL patients. Healthy control subjects matched to gender and age were included, with respect to the well-known decline of sexual health with age. ${ }^{10}$ Sexual function was examined by a self-rating scale that quantifies major aspects of sexuality, including physical as well as psychological aspects of sexual functions. 


\section{Methods}

The study was conducted in 2014 at the outpatient units of botulinum neurotoxin (BoNT) treatment at the Departments of Neurology and Ophthalmology at the University Hospital of Bonn. Patients were asked to participate in the study by their attending physicians (MM, BW, SP). Reviews of inclusion and exclusion criteria for patients and controls, and all study examinations, were conducted by the same physicians (MM for $\mathrm{CD}$ and $\mathrm{BW}$ for $\mathrm{BL}$ ). Controls were recruited by $\mathrm{MM}, \mathrm{BW}$, and SP.

\section{Patients and controls}

$\mathrm{CD}$ and BL patients were recruited consecutively. Inclusion criteria were primary $\mathrm{CD}$ or $\mathrm{BL}$ with adult disease onset. Patients eligible for study participation were invited to participate in an examination on sexual dysfunction. Study completion was determined at 100 patients asked to participate in each disease group. In patients willing to participate, exclusion criteria were then reviewed by interviews and clinical examinations. Exclusion criteria were 1) dystonia exceeding focal, multifocal, or cranial segmental distribution (ie, generalized dystonia), 2) any other neurological diseases (such as neuropathy, brain trauma, stroke, PD, hereditary ataxia), 3) affective or schizophrenic psychosis, 4) cognitive impairment (Mini Mental State Examination $[\mathrm{MMSE}]^{11}<27$ ), or 5) ablation of a sexual organ. In all patients, to minimize potential effects on study parameters, examinations were carried out at the end of a standard BoNT treatment interval of at least 12 weeks.

Controls were recruited from the study authors' relatives, acquaintances, and hospital personnel. Spouses or affiliates of the hospitals' patients were not considered as control subjects for the study. Inclusion criterion was adulthood. In persons eligible for study participation, age and sex were noted and they were invited to participate in an examination on sexual dysfunction. In controls willing to participate, exclusion criteria were then reviewed. Controls were excluded if they had been diagnosed with 1) any neurological disease, 2) affective or schizophrenic psychosis, or 3) ablation of a sexual organ. Recruitment of control subjects was independent from the matching process, which was conducted after determination of the patient samples. Control subjects were arranged according to sex and grouped to age ranges of 5 years. Controls to be included as study population were determined by random sampling in regard to sex and age ranges of both patient groups.

\section{Clinical examinations}

Medical history was reviewed in each patient in a semistructured interview. Collected data included age at onset of dystonia, disease duration, and concomitant diseases and medication. Clinical investigation comprised neurological examination, MMSE, and quantification of dystonia (Tsui score $^{12}$ in CD and Blepharospasm Disability Index ${ }^{13}$ in BL). Symptoms of depression were measured by Beck's Depression Inventory-II (BDI-II) ${ }^{14}$ a self-rating form scoring from 0 to 63 . According to the German National Disease Management Guideline for depression, BDI-II score was evaluated as follows: $<10$ no depression; 10-19 mild depressive syndrome; 20-29 moderate depressive syndrome; $\geq 30$ severe depression. Paper files containing study data were numbered consecutively and did not contain any information to allow patient identification (ie, name). Study data were transferred to a database file, carrying over the patient's or control's study number.

\section{Sexual dysfunction}

All questions pertaining to the patients' sexuality were asked in written format, using questionnaires numbered in accordance to the study files. Patients completed the questionnaires by oneself, in a room detached from the outpatient unit, and placed them in a locked urn. Controls used the same questionnaires, numbered accordingly, and completed them at home. They were sent in anonymized envelopes and placed in the urn by one of the authors (SP). The urn was opened at recruitment closure. Data from the questionnaires were transferred to the database according to the study numbers.

To evaluate sexual dysfunction, we used the Arizona Sexual Experience Scale (ASEX), a validated five-item selfrating scale that quantifies five major aspects of sexuality: 1) sex drive, 2) sexual arousal, 3) vaginal lubrication (in females) or penile erection (in males), 4) ability to reach orgasm, and 5) satisfaction from orgasm. ${ }^{15}$ Each item scores from 1 to 6 , adding to a sum score of 5-30, and higher results indicate more sexual dysfunction. A sum score of $\geq 19$, any one item with a score of 5 , or any three items with a score of 4 , respectively, have been established as indicators of sexual dysfunction. Also, in the questionnaire, we asked two questions pertaining to personal status, and the patient's global impression of her or his sexual function: 1) "Do you live in a steady relationship?" (possible answers "yes" or "no"); 2) "Has your sexual function worsened due to dystonia?" (possible answers "yes" or "no").

\section{Statistics}

Statistical analyses were performed using SPSS statistics 22 software (IBM Corporation, Armonk, NY, USA). $P$-values $<0.05$ were considered significant. For group comparisons, we used Students' $t$-test and ANOVA with 
Bonferroni post hoc test (normally distributed samples were expected). To analyze differences in frequencies, we performed chi-squared tests. If the cell size of the contingency table was $<5$, Fisher's exact test was used. Analysis relationship between variables was calculated by binary logistic regression.

\section{Ethics}

The ethics committee of the University Hospital of Bonn (vote 156/07) approved the study. All patients gave written informed consent.

\section{Results}

\section{Patient and control samples}

Study completion was after 4 months, wherein $101 \mathrm{CD}$ patients and $110 \mathrm{BL}$ patients were asked to participate in the study. In all, 116 matched controls were found. In the CD patients, as well as in the control subjects, $68 \%$ agreed to participate in the study, while participation was significantly lower in the BL patients ( $52 \%$ vs $68 \%, P=0.01)$. Seven patients and four control subjects fulfilled the exclusion criteria or had incomplete questionnaires (Table 1).

Sixty-five patients with CD and 54 patients with BL completed the study. Most were male (61\%) and mean age was 59.6 \pm 12.1 years. Age and sex distribution did not differ between patient samples and control subjects. However, compared to BL patients, $\mathrm{CD}$ patients were significantly younger at onset of disease $(43.3 \pm 12.8$ vs $53.4 \pm 11.8$ years $)$ and had a longer disease duration (15.2 \pm 10.2 vs $7.6 \pm 5.6$ years). Overall, depressive symptoms as measured by BDI-II were comparable in both patient groups, while controls scored significantly lower (4.9 \pm 4.4 vs $7.9 \pm 6.9, P=0.004)$. With regard to the severity of depressive symptoms, the prevalence of a mild disturbance of mood (BDI-II score 10-19) was similar in both patient groups and the control subjects. However, moderate or more severe depressive symptoms (BDI-II score $\geq 20$ ) were absent in controls, and significantly more frequent in CD patients $(9 \%, P=0.01)$, yet not in $\mathrm{BL}$ patients. Twelve patients of both samples $(10 \%)$, and one person of the control sample, were on antidepressant medication $(P=0.04)$ (Table 1). Three patients were on trihexiphenidyl (and none of the control subjects), one of which had a pathological ASEX score (not significant). Likewise, frequency of other medications potentially affecting sexual functions (eg, beta blocking agents) did not differ in both patient groups and the control subjects, and did not influence the ASEX results.

\section{Sexual dysfunction}

Sexual dysfunction as indicated by ASEX was present in 29 CD patients $(45 \%)$, significantly more frequent than in the control sample $(\mathrm{n}=14,24 \%, P=0.009)$. In $\mathrm{BL}$ patients, the prevalence of sexual dysfunction was similar to the CD sample $(\mathrm{n}=21,39 \%)$, and yet failed to reach significance $(P=0.06)$. In accordance, the total ASEX score was highest

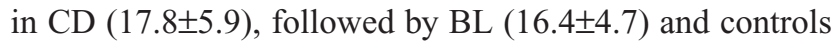
(15.0 \pm 4.2$)$. ANOVA revealed differences between the three groups (one-way ANOVA; F $(2,91)=5.710, P=0.004$ ), while the post hoc test showed significance only when comparing $\mathrm{CD}$ and controls $(P=0.003)$. There were no significant gender differences in the total ASEX score in CD

Table I Study participation and clinical characteristics of cervical dystonia, blepharospasm, and control samples

\begin{tabular}{|c|c|c|c|c|}
\hline & CD & BL & Controls & $P$-value ${ }^{a}$ \\
\hline Invited, n & 101 & 110 & 116 & - \\
\hline Participating, n (\%) & $69(68)$ & $57(52)$ & $79(68)$ & BL vs controls 0.01 \\
\hline Excluded, $\mathrm{n}$ & 4 & 3 & 4 & - \\
\hline Study sample size, $n$ & 65 & 54 & 75 & - \\
\hline Sex (female/male), \% & $4 I-59$ & $35-65$ & $46-54$ & ns \\
\hline Age, years & $58.4 \pm 9.4$ & $60.7 \pm 11.7$ & $59.9 \pm 14.4$ & ns \\
\hline Age at onset, years & $43.3 \pm 12.8$ & $53.4 \pm 11.8$ & - & $\mathrm{CD}$ vs $\mathrm{BL}<0.000 \mathrm{I}$ \\
\hline Disease duration, years & $15.2 \pm 10.2$ & $7.6 \pm 5.6$ & - & $\mathrm{CD}$ vs $\mathrm{BL}<0.000 \mathrm{I}$ \\
\hline Disease severity ${ }^{\mathrm{b}}$ & $9.6 \pm 4.4$ & $1.4 \pm 1.0$ & - & - \\
\hline BDI total ${ }^{\mathrm{c}}$ & $8.2 \pm 7.5$ & $7.5 \pm 6.1$ & $4.9 \pm 4.4$ & $\begin{array}{l}C D \text { vs controls } 0.005 \\
\text { BL vs controls } 0.04\end{array}$ \\
\hline BDI I0-19, n (\%) & $14(2 \mathrm{I})$ & II (20) & $13(17)$ & ns \\
\hline $\mathrm{BDI} \geq 20, \mathrm{n}(\%)$ & $6(9)$ & $3(6)$ & 0 & $C D$ vs controls 0.01 \\
\hline Antidepressants, n (\%) & $7(11)$ & $5(9)$ & $I(I)$ & $\begin{array}{l}C D \text { vs controls } 0.04 \\
\text { BL vs controls } 0.04\end{array}$ \\
\hline
\end{tabular}

Notes: If not stated otherwise, results are given as mean and SD. aNonsignificant results are not listed. ${ }^{\mathrm{b}} \mathrm{CD}$ : Tsui score (range: $\left.0-25\right)$, higher scores indicate more severe dystonia; BL: Blepharospasm Disability Index (range: 0-24), higher scores indicate greater disability. 'BDI-II score (range: 0-63), higher scores indicate more depressive symptoms.

Abbreviations: BL, blepharospasm; BDI, Beck's Depression Inventory; CD, cervical dystonia; n, number; ns, not significant. 
Table 2 Sexual dysfunction in cervical dystonia, blepharospasm, and control samples

\begin{tabular}{|c|c|c|c|c|}
\hline & $C D, n=65$ & BL, $n=54$ & Controls, $n=75$ & $P$-value ${ }^{a}$ \\
\hline $\begin{array}{l}\text { Sexual dysfunction, } n(\%) \\
\text { ASEX total score } \\
\text { ASEX subitems: }\end{array}$ & $\begin{array}{l}29(45) \\
17.8 \pm 5.9\end{array}$ & $\begin{array}{l}21(39) \\
16.4 \pm 4.7\end{array}$ & $\begin{array}{l}18(24) \\
15.0 \pm 4.2\end{array}$ & $\begin{array}{l}C D \text { vs controls } 0.009 \\
B L \text { vs controls ns }(0.06) \\
C D \text { vs controls } 0.003\end{array}$ \\
\hline Sex drive & $3.8 \pm 1.2$ & $3.6 \pm 1.1$ & $3.3 \pm 0.9$ & $C D$ vs controls 0.01 \\
\hline Sexual arousal & $3.6 \pm 1.2$ & $3.2 \pm 1.1$ & $3.0 \pm 0.9$ & CD vs controls 0.001 \\
\hline Vaginal lub/erection & $3.5 \pm 1.3$ & $3.4 \pm 1.1$ & $3.1 \pm 1.1$ & ns \\
\hline Ability to reach orgasm & $3.7 \pm 1.3$ & $3.3 \pm 1.2$ & $3.1 \pm 1.1$ & $C D$ vs controls 0.02 \\
\hline $\begin{array}{l}\text { Satisfaction with orgasm } \\
\text { Worsening of sex, n (\%) } \\
\text { Steady relationship, n (\%) }\end{array}$ & $\begin{array}{l}3.2 \pm 1.5 \\
15(23) \\
49(75)\end{array}$ & $\begin{array}{l}3.1 \pm 1.2 \\
7(13) \\
48(89)\end{array}$ & $\begin{array}{l}2.6 \pm 1.2 \\
- \\
69(92)\end{array}$ & $\begin{array}{l}C D \text { vs controls } 0.01 \\
\text { ns } \\
C D \text { vs controls } 0.007\end{array}$ \\
\hline
\end{tabular}

Notes: If not stated otherwise, results are given as mean and SD. aNonsignificant results are not listed. ASEX (range: 5-30), higher results indicate more sexual dysfunction. Abbreviations: ASEX, Arizona Sexual Experience Scale; BL, blepharospasm; CD, cervical dystonia; lub, lubrication; n, number; ns, not significant.

(one-way ANOVA; F $(1,63)=0.592, P=0.444)$ or BL patients $(\mathrm{F}(1,52)=2.473, P=0.122)$ (Table 2).

Analyses of ASEX subitems uncovered results analogous to the total score, with significant higher scores for sex drive, sexual arousal, ability to reach orgasm, and satisfaction from orgasm when comparing CD patients and controls. In BL patients, no significant differences compared to controls were apparent. Vaginal lubrication/erection scored worse in $\mathrm{CD}$ and $\mathrm{BL}$ patients, yet failed to reach significance. More patients with $\mathrm{CD}(23 \%)$ than $\mathrm{BL}(13 \%)$ reported that dystonia had worsened their sexuality (not significant). In addition, less patients with $\mathrm{CD}(75 \%)$ than with $\mathrm{BL}(89 \%)$, or controls $(92 \%)$, reported a steady relationship $(P=0.007)$.

In the sample of $65 \mathrm{CD}$ patients, logistic regression analyses eliminated variables of dystonia (age of onset, duration, and severity [TSUI score]), as significant predictors of sexual dysfunction (ASEX total score). However, personal status (single, $P=0.04)$, age $(P=0.007)$, and symptoms of depression $(P=0.002)$ were associated to sexual dysfunction in $\mathrm{CD}$. Covariate analyses in logistic regression revealed that the risk of a pathological ASEX score was 4.2 times higher $(B=1,425, P=0.029)$ in the largest subgroup of $C D$ patients with depressive symptoms (mild) compared to CD patients with normal BDI-II. Treatment with antidepressants was not associated with sexual dysfunction in $\mathrm{CD}(P=0.27)$.

\section{Discussion}

Sexual dysfunction as evaluated by ASEX was observed in 50 patients with $\mathrm{CD}$ and BL (42\%), establishing impairment of sexual well-being as a frequent non-motor symptom in adult-onset focal or segmental dystonia. Although the prevalence of sexual dysfunction was considerably lower in the healthy control subjects $(24 \%)$, this discrepancy was significant only in regard to patients suffering from CD, not BL. However, we assume that a meaningful proportion of patients declined to participate in the study, even more so in BL, due to the very personal and potentially embarrassing topic. In particular, this may be the case for patients experiencing regression - or even cessation - of sexual activity. In accordance, willingness to participate in the study was considerably lower (68\% in CD and $52 \%$ in BL patients) when compared to similar examinations in the same patient populations, ranging from $82 \%$ to $95 \% .^{16,17}$ Taken together, we believe that many patients affected by an impairment of sexual well-being, especially with BL, were not included in the study, and that prevalence in dystonia is even higher than determined here.

Our observations confirm those of an earlier study of sexual dysfunction in dystonia based on smaller patient samples $(\mathrm{CD}=30, \mathrm{BL}=30) .{ }^{9}$ Although a different self-rating scale of sexual function was applied, and total prevalence rates were not reported, both patient groups had worse sexual functioning than healthy matched controls, in accordance to our results. Furthermore, risk factors for sexual dysfunction were congruent, most importantly symptoms of depression (and anxiety, which was not examined in our study). Depression is frequent in $\mathrm{CD}$ and $\mathrm{BL},{ }^{4-6}$ and is a well-known major predictor of sexual dysfunction. ${ }^{18,19}$ Even more importantly, patients with dystonia, especially CD, often suffer from stigmatization, ${ }^{7}$ embarrassment, and social phobia. ${ }^{20}$ Selfperceived stigma can be a major source of disability and might deteriorate factors such as self-confidence and body image, crucial for sexual well-being. Furthermore, stigma and social withdrawal affect personal status, explaining the higher portion of singles in our CD patients, in itself associated with sexual dysfunction.

Detailed examination of the various domains of sexual functions revealed impairment in all subitems of ASEX in 
CD patients, with the exception of vaginal lubrication or erection. This observation strengthens the assumption that organic (primary) causes for sexual dysfunction are less important in dystonia. In accordance, genitourinary symptoms were not reported in a study of autonomic imbalance in $85 \mathrm{CD}$ patients, ${ }^{16}$ although sexual functions were not asked for explicitly. Interestingly, neither severity of CD nor age of onset, or disease duration, were associated to sexual dysfunction, refuting that sexuality is compromised directly by motor symptoms of the disorder (secondary). While our observations clearly emphasize that psychosocial (tertiary) mechanisms of sexual dysfunction are paramount in dystonia, we also identified higher age as a predictor of sexual impairment in CD. Many studies demonstrated that with age, prevalence of sexual activity declines and genital dysfunction increases. ${ }^{10}$ As such, while the ASEX does not depict the frequency of sexual intercourses, or cessation thereof, we have no clear information about the subgroup of dystonia patients without any sexual activity. In those, the prevalence of genital dysfunction such as impotence, or reduced vaginal lubrication, might be considerably higher.

\section{Limitations}

We are aware of several limitations of our study. First of all, any examination of sexual functions is hampered by the very subjective definition of sexual well-being, and personal views bias interpretation. Also, many more factors exert an influence on an individual's sexuality that we were unable to address in this study, such as nature of relationship, a patient's bodily and psychological self-concept, and the potential burden of the spouse or caregiver. Furthermore, besides a potential selection bias due to patient recruitment and divergent participation quota as described above, our sample size might restrict generalization of our findings. Although we are not aware of any larger, controlled examination of sexual dysfunction in dystonia, the number of patients included still limits some subgroup analyses, especially those of gender differences, and details of sexual functions as documented by ASEX. This scale has proven substantial internal consistency and construct validity in a variety of neuropsychiatric populations, such that it may be considered a "gold standard." 21 Nevertheless, we must emphasize that self-rating forms deliver global information outlining a health problem, but do not compensate for detailed interviews and clinical examinations. This also applies to BDI, and we might have missed subjects who would have been diagnosed with a relevant mood disorder by a more detailed psychiatric evaluation. Nevertheless, our results reliably outline a health problem in patients with dystonia that clearly warrants further examinations. Given the strong association to depression which was also identified as an important predictor of other non-motor symptoms in dystonia, such as anxiety, ${ }^{6,20}$ sleep disorders, ${ }^{17}$ and autonomic dysfunction ${ }^{16}$ - future studies should, foremost, address therapeutic interventions for mood disorders in patients with dystonia. At the moment, there is no systematic evaluation of pharmacological treatment, or psychological support, for patients with dystonia suffering from depression, anxiety, or stigmatization.

\section{Conclusion}

In conclusion, given a prevalence of $45 \%$, sexual dysfunction represents an important non-motor symptom in CD patients. It is perceived as a burden by the patients, and $23 \%$ ascribe worsening of their sexual life to dystonia. Depression is the most important associated feature. Sexual dysfunction severely impairs quality of life and has to be addressed in the treatment of patients with dystonia.

\section{Acknowledgments}

The study was supported by an unrestricted research grant by Ipsen Pharma, Germany: non-motor comorbidity in cervical dystonia (contract number 11957). An abstract of this paper was presented at the Fifteenth International Congress of Parkinson's Disease and Movement Disorders 2011 as a poster presentation with interim findings. The poster's abstract (number 671) was published in Mov Disord. 2011;26(Suppl 2):S1-S385.

\section{Disclosure}

Dr Marcus Grobe-Einsler has received travel grants and congress fees from Novartis Pharma, Germany. Dr Janis Rebecca Bedarf has received travel grants and honoraria for scientific presentations from Ipsen Pharma, Germany and Merz Pharmaceuticals, Germany. She has also received financial support from PD Fonds Deutschland $\mathrm{gGmbH}$, outside the submitted work. Dr Bettina Wabbels received travel and research grants, and honoraria for scientific presentations from Allergan, Germany; Desitin, Germany; and MERZ Pharmaceuticals, Germany, outside the submitted work. Dr Sebastian Paus reports personal fees from Allergan, Germany; Ipsen Pharma, Germany; and Merz Pharmaceuticals, Germany, outside the submitted work. Milena Marek reports no conflicts of interest in this work.

\section{References}

1. Rees PM, Fowler CJ, Maas CP. Sexual function in men and women with neurological disorders. Lancet. 2007;369(9560):512-525.

2. Meco G, Rubino A, Caravona N, Valente M. Sexual dysfunction in Parkinson's disease. Parkinsonism Relat Disord. 2008;14(6):451-456. 
3. Gündel H, Wolf A, Xidara V, et al. High psychiatric comorbidity in spasmodic torticollis: a controlled study. J Nerv Ment Dis. 2003; 191(7):465-473.

4. Fabbrini G, Berardelli I, Moretti G, et al. Psychiatric disorders in adultonset focal dystonia: a case-control study. Mov Disord. 2010;25(4): $459-465$.

5. Wenzel T, Schnider P, Wimmer A, Steinhoff N, Moraru E, Auff E. Psychiatric comorbidity in patients with spasmodic torticollis. $J$ Psychosom Res. 1998;44(6):687-690.

6. Gündel H, Wolf A, Xidara V, Busch R, Ceballos-Baumann AO. Social phobia in spasmodic torticollis. JNeurol Neurosurg Psychiatry. 2001;71(4):499-504.

7. Rinnerthaler M, Mueller J, Weichbold V, Wenning GK, Poewe W. Social stigmatization in patients with cranial and cervical dystonia. Mov Disord. 2006;21(10):1636-1640.

8. World Association for Sexual Health. Sexual Health for the Millennium. A Declaration and Technical Document. Washington, DC: World Association for Sexual Health; 2008.

9. Perozzo P, Salatino A, Cerrato P, Ricci R. Sexual Well-Being in Patients with Blepharospasm, Spasmodic Torticollis, and Hemifacial Spasm: A Pilot Study. Front Psychol. 2016;7:1492.

10. Lindau ST, SchummLP, LaumannEO, Levinson W, O'MuircheartaighCA, Waite LJ. A study of sexuality and health among older adults in the United States. N Engl J Med. 2007;357(8):762-774.

11. Crum RM, Anthony JC, Bassett SS, Folstein MF. Population-based norms for the Mini-Mental State Examination by age and educational level. JAMA. 1993;269(18):2386-2391.

12. Tsui JK, Eisen A, Stoessl AJ, Calne S, Calne DB. Double-blind study of botulinum toxin in spasmodic torticollis. Lancet. 1986;2(8501): $245-247$.
13. Lindeboom R, De Haan R, Aramideh M, Speelman JD. The blepharospasm disability scale: an instrument for the assessment of functional health in blepharospasm. Mov Disord. 1995;10(4):444-449.

14. Kühner C, Bürger C, Keller F, Hautzinger M. [Reliability and validity of the Revised Beck Depression Inventory (BDI-II). Results from German samples]. Reliabilität und Validität des revidierten Beck-Depressionsinventars (BDI-II). Nervenarzt. 2007;78(6):651-656. German.

15. McGahuey CA, Gelenberg AJ, Laukes CA, et al. The Arizona Sexual Experience Scale (ASEX): reliability and validity. J Sex Marital Ther. 2000;26(1):25-40.

16. Hentschel F, Dressler D, Abele M, Paus S. Impaired heart rate variability in cervical dystonia is associated to depression. JNeural Transm. 2017;124(2):245-251.

17. Paus S, Gross J, Moll-Müller M, et al. Impaired sleep quality and restless legs syndrome in idiopathic focal dystonia: a controlled study. J Neurol. 2011;258(10):1835-1840.

18. Seidman SN, Roose SP. Sexual dysfunction and depression. Curr Psychiatry Rep. 2001;3(3):202-208.

19. Williams K, Reynolds MF. Sexual dysfunction in major depression. CNS Spectr. 2006;11(8 Suppl 9):19-23.

20. Ozel-Kizil ET, Akbostanci MC, Ozguven HD, Atbasoglu EC. Secondary social anxiety in hyperkinesias. Mov Disord. 2008;23(5):641-645.

21. Byerly MJ, Nakonezny PA, Fisher R, Magouirk B, Rush AJ. An empirical evaluation of the Arizona sexual experience scale and a simple one-item screening test for assessing antipsychotic-related sexual dysfunction in outpatients with schizophrenia and schizoaffective disorder. Schizophr Res. 2006;81(2-3):311-316.
Neuropsychiatric Disease and Treatment

\section{Publish your work in this journal}

Neuropsychiatric Disease and Treatment is an international, peerreviewed journal of clinical therapeutics and pharmacology focusing on concise rapid reporting of clinical or pre-clinical studies on a range of neuropsychiatric and neurological disorders. This journal is indexed on PubMed Central, the 'PsycINFO' database and CAS,

\section{Dovepress}

and is the official journal of The International Neuropsychiatric Association (INA). The manuscript management system is completely online and includes a very quick and fair peer-review system, which is all easy to use. Visit http://www.dovepress.com/testimonials.php to read real quotes from published authors. 